\title{
Real-time control of divertor detachment in H-mode with impurity seeding using Langmuir probe feedback in JET-ITER-Like Wall
}

\author{
C. Guillemaut ${ }^{1,2}$, M. Lennholm ${ }^{3}$, J. Harrison ${ }^{4}$, I. Carvalho ${ }^{2}$, D. Valcarcel', R. Felton ${ }^{4}$, \\ S. Griph ${ }^{4}$, C. Hogben ${ }^{4}$, R. Lucock ${ }^{4}$, G. Matthews ${ }^{4}$, C. Perez Von Thun ${ }^{5}$, R. Pitts ${ }^{6}$, S. Wiesen ${ }^{5}$ \\ ${ }^{1}$ EUROfusion Consortium, JET, Culham Science Centre, Abingdon, OX14 3DB, UK \\ ${ }^{2}$ Instituto de Plasmas e Fusão Nuclear, Instituto Superior Técnico, Universidade de Lisboa, P-1049- \\ 001, Lisboa, Portugal \\ ${ }^{3}$ European Commision, B-1049 Brussels, Belgium \\ ${ }^{4}$ CCFE, Culham Science Centre, Abingdon OX14 3DB, UK \\ ${ }^{5}$ Forschungszentrum Jülich GmbH, Institut für Energie- und Klimaforschung - Plasmaphysik, 52425 \\ Jülich, Germany \\ ${ }^{6}$ ITER Organization, Route de Vinon CS 90 046, 13067 Saint-Paul-Lez-Durance, France
}

\begin{abstract}
DT discharges with $500 \mathrm{MW}$ of fusion power on ITER will rely on partially detached divertor operations to keep target heat loads at manageable levels. Such divertor regime will be maintained by a real-time control system using the seeding of radiative impurities like nitrogen $(\mathrm{N})$, neon or argon as actuator and one or a combination of diagnostics signals as feedback. Recently, real-time control of divertor detachment has been successfully achieved in Type I ELMy H-mode JETITER-like Wall discharges by using saturation current $\left(I_{\text {sat }}\right)$ measurements from divertor Langmuir probes as feedback signal to control the $\mathrm{N}$ seeding. The level of divertor detachment is calculated in real-time by comparing the outer target peak $I_{\text {sat }}$ measurements to the peak $I_{s a t}$ value at the roll-over in order to control the opening of the $\mathrm{N}$ injection valve. Real-time control of detachment has been achieved in fixed and swept strike point experiments. The system has been progressively improved and can now automatically drive the divertor conditions from attached through high recycling and roll-over down to a user-defined level of detachment. Such demonstration is a successful proof of principle in the context of ITER.
\end{abstract}

\section{Introduction: necessity of detachment real-time control on ITER}


DT discharges with $500 \mathrm{MW}$ of fusion power on ITER will rely on partially detached divertor operations to keep target heat loads at manageable levels [1]. These experiments will rely on a Type I ELMy H-mode and will be achieved with a tungsten $(\mathrm{W})$ divertor and a beryllium $(\mathrm{Be})$ main chamber wall. Since $\mathrm{W}$ and $\mathrm{Be}$ are very poor radiators in the scrape-off layer (SOL), the seeding of impurities like nitrogen $(\mathrm{N})$, neon (Ne) or argon ( $\mathrm{Ar}$ ) can be used to dissipate significant amounts of power radiatively. In ITER, 60-70 \% of the SOL power must be radiated to keep the divertor target power density around $10 \mathrm{MW}^{-2}$, see [2]. Such level of power dissipation will cool the target plasma down to low electron temperatures which will trigger a combination of recombination and transport processes [3,4] resulting in the roll-over and reduction of target particle flux. The divertor detachment regime will be maintained by a real-time control system using the seeding of radiative impurities as actuator and one or a combination of real-time diagnostic measurements as feedback signals. Since Langmuir probes (LP) are expected to be part of the ITER divertor diagnostics, there will be the possibility to achieve control of the target power density through the control of the detachment level by using the saturation current $\left(I_{\text {sat }}\right)$ measurements. The level of divertor detachment can be calculated in real-time by comparing the outer target (OT) peak $I_{\text {sat }}$ measurements to the peak $I_{\text {sat }}$ value at the roll-over in order to control the opening of the impurity gas injection valve.

The JET-ITER-like Wall (ILW) [5] comprises a W divertor and Be main chamber wall thus matching the material configuration planned for ITER. In order to provide a proof of principle for ITER, real-time detachment control with $\mathrm{N}$ seeding and LP feedback has been attempted in several dedicated JET-ILW Type I ELMy Hmode experiments. The discharges have been achieved with a toroidal field of $2.2 \mathrm{~T}$, a plasma current of $2 \mathrm{MA}, 8 \mathrm{MW}$ of neutral beam injection (NBI) heating and $2 \mathrm{MW}$ of ion cyclotron resonance heating $(\mathrm{ICRH})$. The divertor configuration featured a vertical inner target with a horizontal OT and the detachment real-time control system was using 8 of the OT LP, see red squares in Fig. 1. The system is described in details in Section 2 and has gradually evolved in complexity from the first experimental attempts (Section 3) to become a nearly fully automatized system in the most recent experiments (Section 4).

\section{Description of the detachment real-time control system on JET}


The divertor LP are placed at 36 poloidal locations in 3 different modules, dee Fig. 1. In real-time detachment control experiments, they are operated in saturation current with a constant voltage bias at $\sim-150 \mathrm{~V}$ interrupted every $20 \mathrm{~ms}$ with a brief excursion at $\sim 5 \mathrm{~V}$ (see Fig. 2) to prevent arcing damage. The detachment control system presented here can use up to 16 simultaneous Langmuir probe signals in real-time. In the experiments described here, 8 signals have been used from the outboard probes of Tile 5 . As shown in the diagram in Fig. $3, I_{\text {sat }}$ measurements are sent to the UXD7 data acquisition unit with a sample frequency of $100 \mathrm{kHz}$. The signals are first digitalized and then momentarily stored in random access memory (RAM) during the discharge before being permanently saved as files after the discharge.

As shown in Fig. 3, the generation of LP real-time data requires the use of a new channel involving a field programmable gate array (FPGA) to filter and downsample the $I_{\text {sat }}$ data to a much lower frequency in order to be handled by the realtime network. The latter cannot work with a sampling rate higher than $250 \mathrm{~Hz}$. The FPGA is used as a low-pass filter with a cut frequency set at $50 \mathrm{~Hz}$ to eliminate the positive voltage "blips" and the ELMs. The filtered signal is averaged over $20 \mathrm{~ms}$ periods which reduces the $I_{\text {sat }}$ sampling rate down to $50 \mathrm{~Hz}$. The resulting real-time $I_{\text {sat }}$ corresponds to inter-ELM conditions (Fig. 4) and is fast enough compared to the $\sim 1 \mathrm{~s}$ response time of the $\mathrm{N}$ injection system. The real-time signals are first sent on a private Ethernet network where the channels can be configured in a Linux PC before being transferred to the general JET real-time control network. Then, the $I_{s a t}$ signals can be used by the real-time central controller (RTCC). At any time $t$ during the discharge, the RTCC creates an $I_{s a t}$ value from the peak saturation current found amongst the 8 real-time signals coming from the red probes in Fig. 1 and compares it to the peak $I_{\text {sat }}$ at roll-over $I_{\text {roll }}$ by calculating the attachment fraction $I_{\text {sad }} / I_{\text {roll }}$.

All real-time detachment control experiments start with an attached plasma. In the first phase of the discharge the $\mathrm{N}$ seeding is controlled to reach the roll-over at a time $t_{\text {roll }}$ when:

$$
\max \left(\mathrm{I}_{\text {sat }}\left(\mathrm{t}_{\text {roll }}\right)\right)=I_{\text {roll }} \text {, }
$$


and then the controller continues the $\mathrm{N}$ seeding until the attachment fraction $R e f$ requested before the discharge is achieved such as:

$\frac{\max \left(\mathrm{I}_{\text {sat }}(\mathrm{t})\right)}{I_{\text {roll }}}=\operatorname{Ref}$

To do so, the RTCC calculates a real-time Error as follows:

$\operatorname{Error}(t)=\operatorname{Ref}-\frac{I_{\text {sat }}(t)}{I_{\text {roll }}}$

in order to obtain the percentage of aperture $A(t)$ of the $\mathrm{N}$ injection valve using the gains $G$ and the time constant $\tau$ as follows:

$$
A(t)=G\left(\operatorname{Error}(t)+\frac{1}{\tau} \int_{0}^{t} \operatorname{Error}\left(\mathrm{t}^{\prime}\right) d t^{\prime}\right)
$$

where $t^{\prime}$ is the time variable of integration.

$G$ controls the sensitivity of the controller to the feedback signal: the higher the gains, the higher the sensitivity. $\tau$ weights the integral term (second term) compared to the proportional term (first term) and has been kept constant at $\tau=2 \mathrm{~s}$. In the most basic version of the controller, $I_{\text {roll }}$ and $G$ are set before the discharge by the user (see Section 3 below) while more advanced controllers can calculate these parameters in real-time (see Section 4 below).

\section{First experimental detachment control attempts with basic controller}

\subsection{Fix strike point}

The first attempt of detachment real-time control with LP feedback in JET-ILW Hmode discharges has been achieved with fix outer strike point, see Fig. 1. The values for $I_{\text {roll }}$ and $G$ were set before the experiments using data from previous reference detachment discharges without controller. In the first attempt, in discharge 
\#89746, an increase of $I_{\text {sal }} I_{\text {roll }}$ from 0.5 to 1 was programmed before the roll-over and once $I_{\text {sat }}=0.8 I_{\text {roll }}$, the controller switched to a required attachment ratio decreasing linearly for $1.5 \mathrm{~s}$ and then kept stable at $R e f=0.5$. The progressive linear decrease of the attachment ratio was necessary to avoid too strong $\mathrm{N}$ seeding rates which could have brought the level of detachment lower than the desired value. As shown in Fig. $5 \mathrm{a}$, in discharge \#89746, the requested $50 \%$ reduction of peak $I_{\text {sat }}$ (from roll-over) has been achieved at $11 \mathrm{~s}$ and maintained for $3 \mathrm{~s}$ until the end of the discharge at $14 \mathrm{~s}$.

As shown in Fig. 5b, a second successful attempt of real-time detachment control has been achieved in \#89751 with $75 \% I_{\text {sat }}$ reduction of peak $I_{\text {sat }}$ (from rollover) maintained for the last $\sim 2 \mathrm{~s}$ of the discharge. In both discharges \#89746 and \#89751, the level of detachment of the OT plasma appears to be very sensitive to the $\mathrm{N}$ seeding rate just after the roll-over and less sensitive once the required partially detached steady-state is reached. Therefore, $G$ should ideally be variable and have a low value around the roll-over and a high value once the requested detachment level is nearly reached. In \#89746 and \#89751, $G$ was set high at a constant value to facilitate the stabilization of the partially detached phase with the risk of generating oscillations in the $I_{\text {sal }} / I_{\text {roll }}$ signal after the roll-over, like in Fig. $5 \mathrm{~b}$.

\subsection{Swept strike point}

For target power load spreading, it is sometimes necessary to sweep the strike point between different stakes of Tile 5 (Fig. 1) during JET-ILW operations and it may also be a possibility for ITER. That is why, further real-time detachment control experiments were attempted with swept outer strike point in JET-ILW H-mode discharges. In discharges \#89755 and \#89756, the outer strike point was swept between stake $\mathrm{C}$ and $\mathrm{D}$ (Fig. 1) at a frequency of $4 \mathrm{~Hz}$ and $G$ was kept constant, similarly as in the experiments described in Section 3.1.

Since the OT peak $I_{\text {sat }}$ moves from one probe to another during the sweep, it is not measured in between probes on Tile 5 (Fig. 1). Therefore, the averaging done after the low-pass filtering in the FPGA reduces the measured roll-over $I_{\text {sat }}$ to $\sim 70-$ $80 \%$ of the reference $I_{\text {roll }}$ obtained from fix strike point detachment experiments. Thus, it was necessary to adapt the controller to switch from pre-roll-over requirement to post-roll-over requirements (Fig. $6 \mathrm{a}$ and b) when $I_{\text {sat }}=0.5 I_{\text {roll }}$. 
In \#89755 and \#89756, the requested peak $I_{\text {sat }}$ reductions from roll-over were $75 \%$ and $50 \%$, respectively. In \#89756, the modest $I_{\text {sat }}$ reduction placed the OT plasma in conditions where $I_{\text {sal }} / I_{\text {roll }}$ is still very sensitive to $\mathrm{N}$ seeding. That is why the high gain which is suitable for \#89755 is responsible for strong oscillations in \#89756, see Fig. $6 a$ and $b$. It is however a clear evidence that the real-time detachment control system based on LP feedback works and is very sensitive to divertor target conditions. None the less, a more automatized system would be required to be relevant for JET-ILW and ITER operations.

\section{Automatization of the detachment real-time controller}

\subsection{Automatic $I_{\text {sat }}$ roll-over search}

The peak $I_{\text {sat }}$ at roll-over is not necessarily known in JET-ILW as well as in ITER. The controller should not have to rely on previous reference detachment experiments or code predictions and should therefore have the ability to find the roll-over on its own. An upgrade of the system containing this new feature has been tested in the same type of $\mathrm{H}$-mode discharge as previous real-time detachment control experiments.

The main requirement of this more evolved controller is to start the control of the $\mathrm{N}$ injection valve while the OT plasma is still attached. Mention has to be made that the controller gain was still fixed at a constant value before the discharge. In the initial attached phase, the controller runs in feedforward and opens the $\mathrm{N}$ injection valve to the maximum allowed ( $45 \%$ of full aperture). It compares the instantaneous peak $I_{\text {sat }}$ with the maximum reached since the beginning of the controlled period and switches to feedback control once the difference between both values has reached $90 \%$ of the requested $I_{\text {sat }}$ reduction.

This controller has been tested with success in discharge \#90516 where the peak OT $I_{\text {sat }}$ was reduced by $75 \%$ from roll-over, as requested, see Fig. 7. However, the gain of the controller was too high and this is the reason for the very clear oscillations that can be seen in Fig. 7. To avoid this problem, the controller should be able to adapt automatically the gain depending on the OT conditions.

\subsection{Automatic adaptation of the gain}


For a stable, oscillation-free control system, high sensitivity of the plasma to the $\mathrm{N}$ valve aperture just after the roll-over should be compensated by a low controller gain. On the other hand, low plasma sensitivity to $\mathrm{N}$ injection in deep detached conditions should be compensated by a higher gain for the controller. Such features have been implemented in another upgraded controller and tested in JET-ILW H-mode experiments.

Mention has to be made that this development is independent from the automatic roll-over search feature discussed in the previous Section. Both upgrades have been tested separately and have not yet been merged. Thus, it was still necessary to programme a pre-roll-over phase with an $I_{\text {roll }}$ value obtained from previous detachment reference experiments. For automatic gain adaptation, it has been found by fitting $G\left(I_{\text {sat }}\right)$ from previous stable detachment real-time control experiments that $G$ should vary as follows:

$$
G(t)=\exp \left[-1.62\left(\frac{I_{\text {sat }}(\mathrm{t})}{I_{\text {roll }}}-0.5\right)\right]
$$

The successful results from discharges \#90517 and \#90519 shown in Fig. 8a and b have been obtained by varying $G$ according to (5). Respectively, $70 \%$ and $90 \%$ of peak $I_{\text {sat }}$ reduction from roll-over have been achieved as requested in \#90517 and \#90519 without oscillations with this controller.

\section{Conclusions}

Safe ITER divertor operations during DT discharges with $500 \mathrm{MW}$ of fusion power will rely on a partially detached regime which will be maintained by a real-time control system. Since it is planned to install LP in ITER divertor, there will be the possibility to use the $I_{s a t}$ signal to calculate the level of divertor detachment in realtime by comparing the OT peak $I_{\text {sat }}$ measurements to the peak $I_{s a t}$ at the roll-over. Thus, the divertor detachment level can be used to control the opening of the impurity gas injection valve.

In order to provide a proof of principle for ITER, real-time detachment control with $\mathrm{N}$ seeding and LP feedback has been attempted in several dedicated JET-ILW 
Type I ELMy H-mode experiments and achieved with success. The system developed for JET managed to control detachment with fix and swept outer strike point in horizontal OT divertor configuration. It has been shown that the roll-over can be found automatically, providing that the control starts in attached divertor conditions. It has also been demonstrated that the gain of the controller can be automatically adapted in real-time to avoid oscillations of the system.

This system could be useful for JET operations with impurity seeding and could be coupled with other real-time controllers. Given the success of these first attempts, other tests are foreseen in different divertor configuration (vertical targets) and also with other impurities like $\mathrm{Ne}, \mathrm{Ar}$ or $\mathrm{CD}_{4}$. The next trials should involve a fully automatic system capable of driving the divertor plasma from attached conditions down to the requested detached state by coupling the adaptive gain and roll-over search features presented here.

\section{Acknowledgments}

This work has been carried out within the framework of the EUROfusion Consortium and has received funding from the Euratom research. IST activities also received financial support from "Fundação para a Ciência e Tecnologia" through project UID/FIS/50010/2013. The views and opinions expressed herein do not necessarily reflect those of the European Commission.

\section{References}

[1] R.A. Pitts et al., 2011 J. Nucl. Mat. 415 S957-64

[2] R.A. Pitts et al., Phys. Scr. T138 (2009) 014001.

[3] C. Guillemaut et al NF2014

[4] D. Guillemaut et al PSI 2016

[5] G.F. Matthews et al 2011 Phys. Scr. 2011014001 


\section{Figures captions:}

Fig. 1 Horizontal target magnitic configuration in JET-ILW divertor with LP positions (squares). Only the red LP are used by the real-time detachment control system.

Fig. 2 Top: LP voltage wave form used for real-time detachment control; bottom: corresponding $I_{\text {sat }}$ measurements.

Fig. 3 Diagram of the real-time detachment control system with LP feedback.

Fig. 4 Example of LP $I_{\text {sat }}$ measurements (magenta curve) with its corresponding down-sampled and filtered real-time $I_{\text {sat }}$ (blue curve).

Fig. 5 (a) Top: $\mathrm{N}$ injection rate time trace. Bottom: real-time level of attachment (Isat/Iroll) time trace (blue cruve) with pre-roll-over request (green cruve) and postroll-over request (red curve) for discharge \#89746. (b) Same as (a) for discharge \#89751.

Fig. 6 Same as Fig. 5 for (a) discharge \#89755 and (b) discharge \#89756.

Fig. 7 Same as Fig. 6 for discharge \#90516. No pre-roll-over request was necessary since the controller was using the automatic roll-over search feature.

Fig. 8 Same as Fig. 6 for (a) discharge \#90517 and (b) discharge \#90519. 


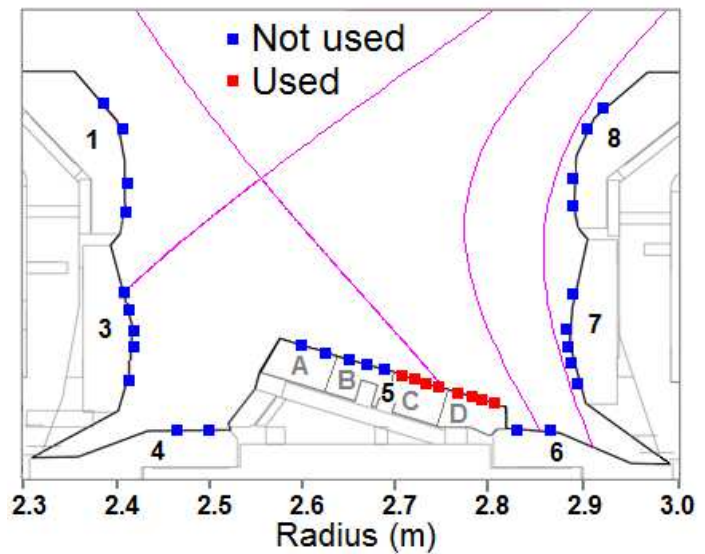

Fig. 1 


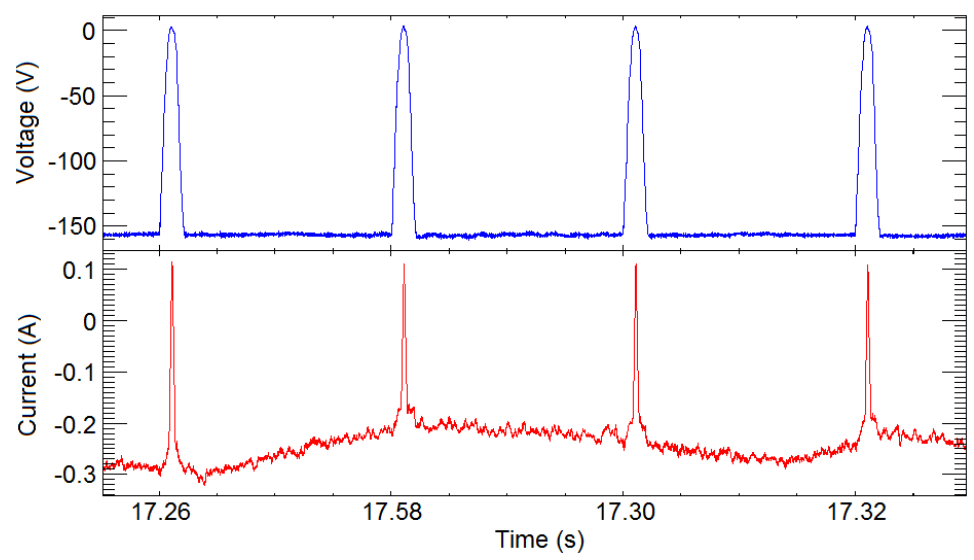

Fig. 2 


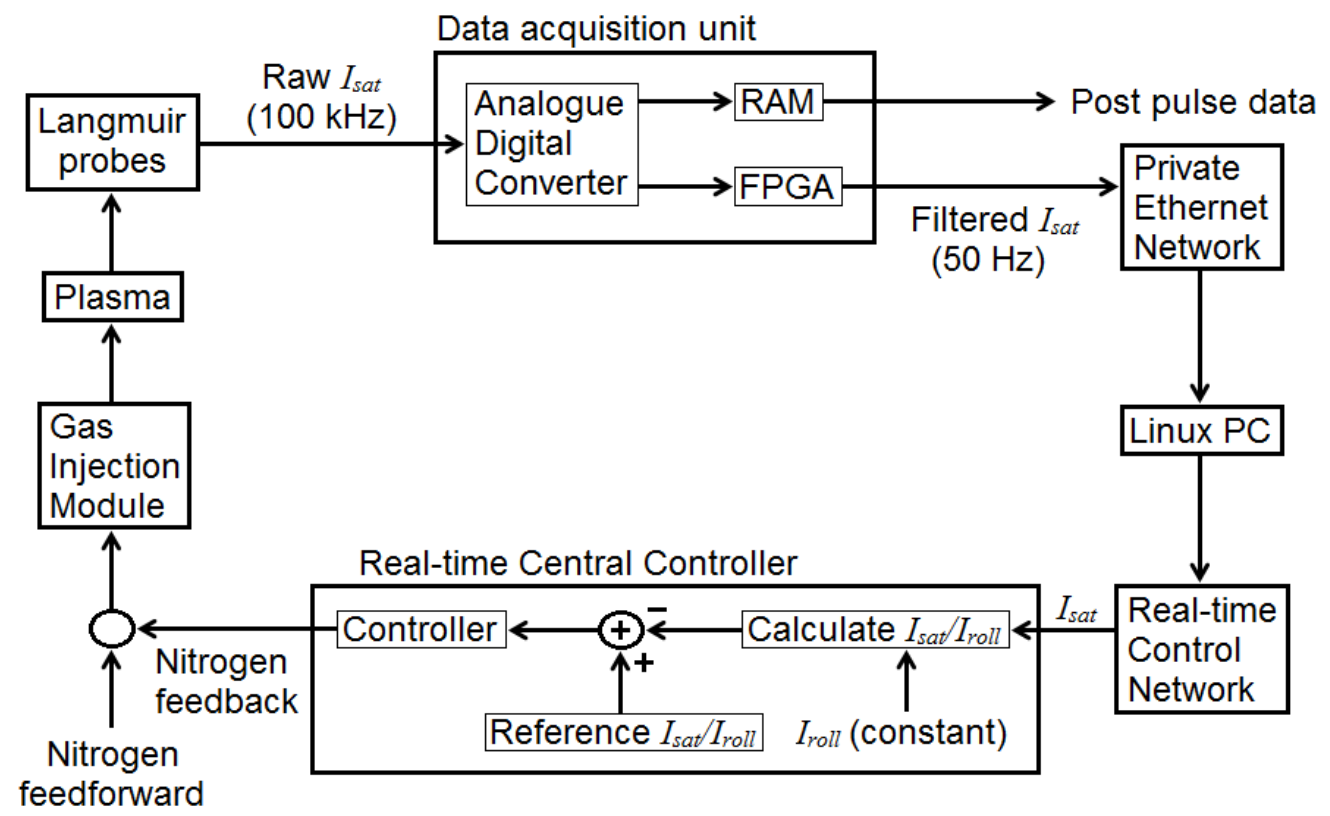

Fig. 3 


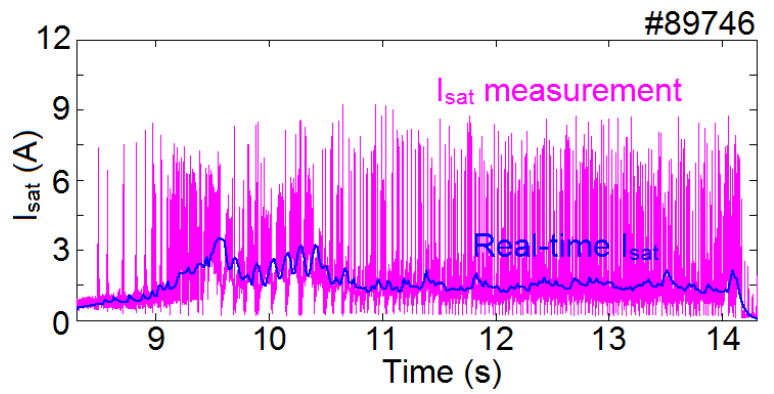

Fig. 4 

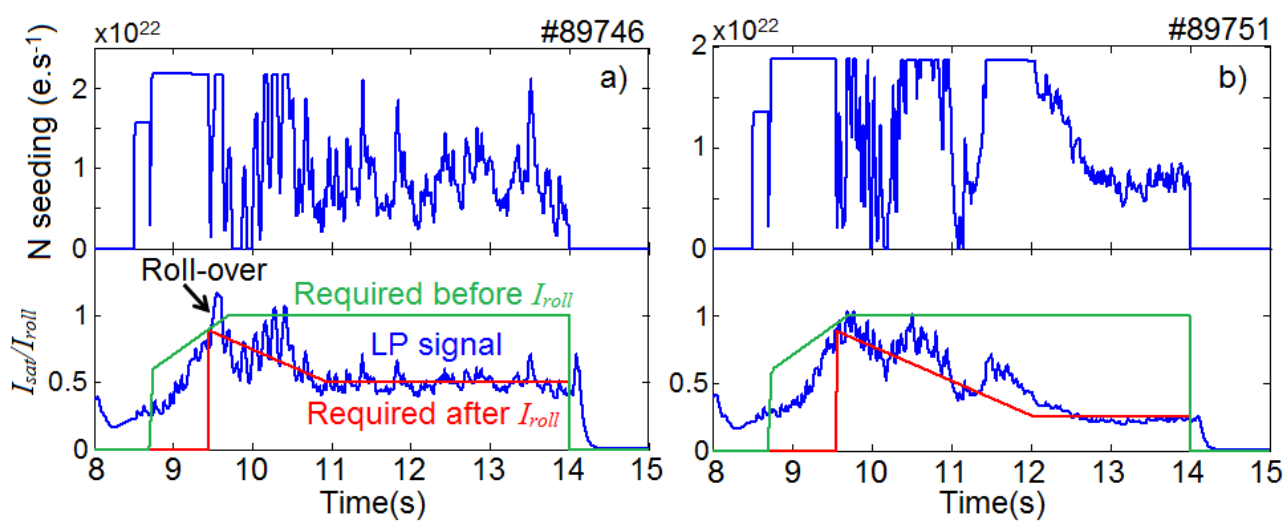

Fig. 5 

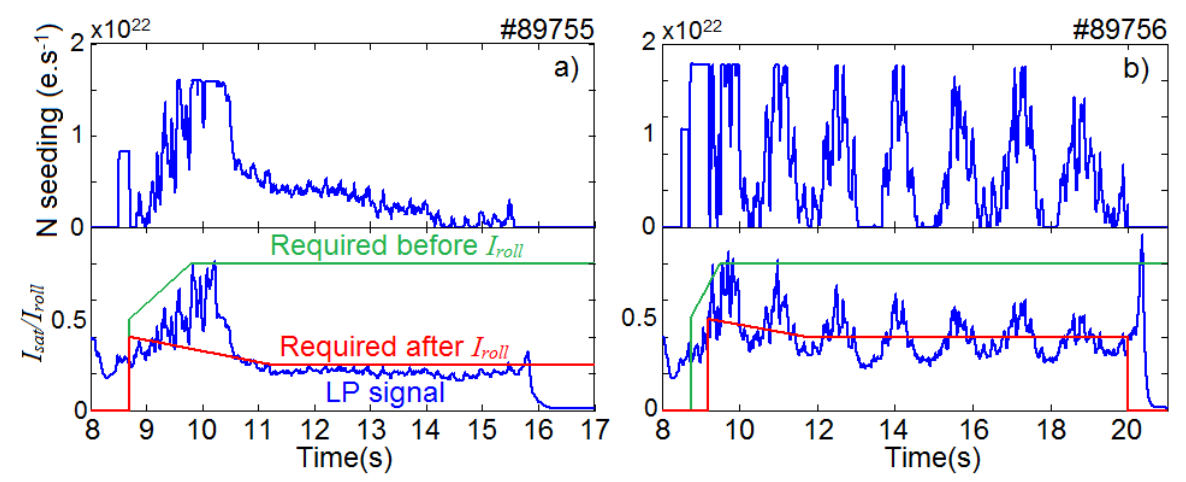

Fig. 6 


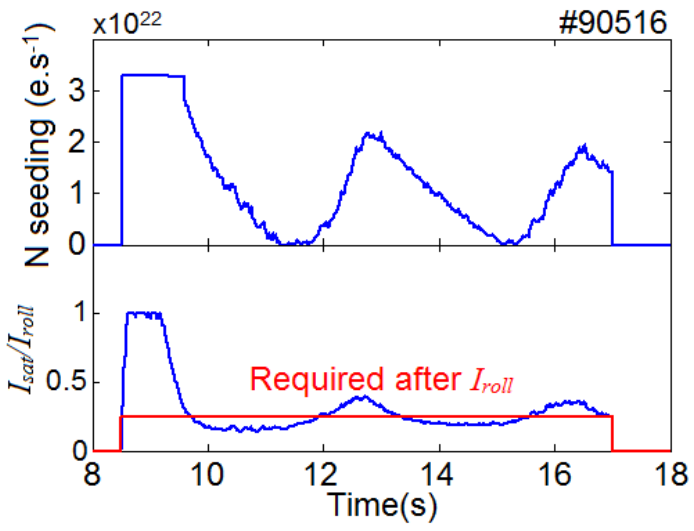

Fig. 7 

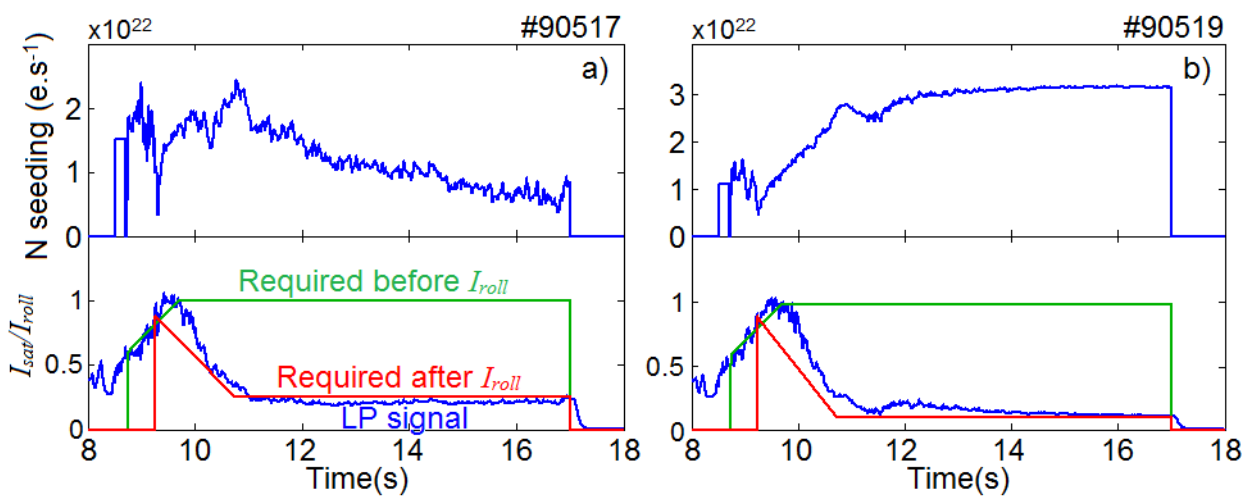

Fig. 8 